\title{
On IPTV Live Video Coding Rate of Explore
}

\author{
Yuanlin Li
}

Television Broadcast control center, Hunan Changsha 410003

Abstract: With the network of development new media of application have been fully integrated into to We of work and life. How to national?1.2BillionIPTVUser provide high quality of image service is Radio and Television New Media has been thinking Study of topic. The author through years of work experience and a large number of experimental data summarize and verify the to adapt to in IPTV live video rate of standard.

Keywords: Coding; H. 264; IPTV; rate; pqr; PSNR; SSIM; Image Quality

With the State Council on three nets fusion policy of call national IPTV industry such as rain have a rapid development. IPTV integrated broadcast control platform the implementation of national unified planning, unified standard, unified organization, unified management.

At present IPTV video coding format has basic unified "with H. 264 as an basic standard with the Communication Operators of broadband Vigorously Development Video Coding rate also will with the conditions of mature and gradually improve. In same coding technology under if rate the greater the quality better? What kind of rate cost highest? How to online test video quality of quality?

In accordance with the First of White Paper on IPTV live coding of standard which coding just general The provisions of the mainstream coding format but at present for most IPTV are "with H. 264 as an coding standard its history and technology reason in this discussed in don't do more than.

Coding rate mode for CBR this point and cable network VBR have nature of the difference. For multi-channel reuse a frequency or a fixed bandwidth under VBR or ABR all has a unique of advantage. And IPTV just instead fixed a bandwidth running a group multicast channel certain is close to full load of best utilization is preferred. So CBR and VBR no which is good, which difference of that just confirmed the word: no absolute only relative of perfect matching.

Channel for everyone general all use Mpeg1L2 or aac hd sometimes also will the Dolby AC3 or EAC3 this to according to local set-top box of license whether support to decision. Sampling Rate are default "with 48 KHZ. Channel standard qing dou will select mono hd to choose stereo or 5.1 .

Video Coding rate as an this discussed of point in the standard even though the have a recommendations range standard clear: $1.5 \sim 2.5 \mathrm{Mbps}$ and HD 1080i: 8 Mbps 720 P: 6 Mbps but no to absolute definition. This actually also can understand should all over the network situation not and IPTV was is a video can do dynamic adjustment of development-oriented platform. This the around of content provider for is confused of place is also can breakthrough of place can not stick to one pattern according to their own situation make best coding the best image quality.

Now we just how to in existing bandwidth conditions under coding the best of quality do test work. Below we the different rate of performance to do the analysis. Test target by special test instrument test the different encoder coding quality.Test equipment are as follows. Test Instrument: Tektronix PQA500; H.264IP solution code the:. Ray IRD2963; 
Switch: cisco2960.

By Tektronix PQA500 a SD and HD-SDI standard image frame sequence after as points will signal distribution the manufacturer H. 264 encoder Harley IRD2963 decoder to IPH.264 flow decoding into SDI signal again to solution out of SDI signal back PQA500 instrument.

PQA500 will to a and receive of image frame synchronous contrast calculation the between the difference the last of $\mathrm{PQR}$ value $0 \sim 10$ score $\mathrm{PQR}=0$ said test sequence and reference sequence completely same $\mathrm{PQR}$ value the greater the injury the greater the degree. $\mathrm{PQR}=1$ almost awareness can't injury; pqr $=3$ have perceived continuous injury but is not strong; $\mathrm{pqr}=10$ obvious perceived injury.

The test have standard clear encoder and HD encoder are mainstream of encoder (Ericsson, Harley, Ateme and) follow-up data for Encoder of average the present out of data not representative coding products, only as an rate characteristics reference.

HD by three types have representative of image frame to complete test:

Duckstakeoff, intotree, parkjoy.

The SD is tested by five representative image frames: windmill, train, soccer, face, table tennis.

Analyze Based on final test data:

In order to get a high cost-effective value at $17.5 \mathrm{Mbps}$, the quality of MPEG2 is greatly improved compared with that of $12 \mathrm{Mbps}$, and it will be slightly inferior to that of $24 \mathrm{Mbps}$.

H. 264 high-definition encoding will get a better quality at $8 \mathrm{Mbps}$, and as the rate increases, the quality will also increase, to $12 \mathrm{Mbps}$, it shows a bottleneck, the image quality from higher bit rate is not obvious. H. $26412 \mathrm{Mbps}$ and MPEG2 17.5 Mbps will have a slight upper hand in addition to dealing with water ripple. This also shows another problem. In the case of high bit rate of MPEG2, it has certain advantages to encode complex images, which is also the user reflection of some game, NBA basketball and other game broadcast, the reason why the network is better than IPTV. But in the general scenario environment, the advantages of high compression ratio of IPTV will also be reflected, so the general TV series and other conventional programs, IPTV image quality still has an advantage.

From the test results, the quality of motion image encoding with rich color is poor, especially in the low bit rate, the damage is quite serious. The feature image processing ability is strong, and the performance of the two kinds of code rate is better. According to the data analysis, we can get a high quality video quality in the case of $2.5 \mathrm{Mbps}$.

At present, it is suitable to use $264 \mathrm{Mbps}$ as the mainstream of standard definition encoding for IPTV with H. 2.5 . In the case of high definition, according to the difference of Urban broadband access, it is generally divided into $8 \mathrm{Mbps}$ and $12 \mathrm{Mbps}$ to provide services. In general, IPTV has better features at such rate.

It is also a major issue to regulate the quality of baseband signal to IP signal in the integrated platform. It is suitable to adopt a PQA-like comparative testing mechanism. Below we test the IPTV all the way HD channel.

Comparative Test on the image quality analysis of primon:

Purpose of the experiment: to verify the real-time scoring function of primon Image Quality

Analysis of the signal quality of Hunan Satellite TV after IPTV encoding.

Test object: Hunan Satellite TV HD SDI signal, H. 264 ts signal after encoding, the rate is $8 \mathrm{Mbit} / \mathrm{s}, \mathrm{H}$. 264 ts signal after encoding, the rate is $12 \mathrm{Mbit} / \mathrm{s}$.

Working principle: primon can detect the PSNR (peak signal-to-noise ratio) and SSIM (Structural similarity) of the two signals in real time to evaluate the quality of the signal after encoding. Where PSNR is

$0 \sim 0 \sim$ Between $100 \mathrm{db}$, the greater the value, the less loss of the screen, usually the value will be $20-20 \sim$ Between 60 . SSIM represents the similarity of the image from three aspects, namely brightness, contrast and structure $\sim$ Between 1 , the greater the value of the image loss is smaller.

Experimental data: according to different types of programs, 8 groups of three groups of encoded signals were compared with the PSNR and SSIM average of the baseband signals before encoding. (See table 3)

Subjective analysis of picture quality: refer to the above two PSNR thermal map screenshots, in which the red part 
of the picture shows that the PSNR is lower and the gray part shows that the PSNR is higher and the loss is lower, it can be seen that there is almost no red color in the two encoded images, and the subjective perception of the image is of high quality. At this time, the $8 \mathrm{~m}-264$ stream and $12 \mathrm{~m}-264$ stream screen are completely gray, and there is almost no loss.

Objective analysis of picture quality: referring to the above PSNR and SSIM table, the average PSNR of $8 \mathrm{~m}-264$ bitstream and baseband signal is $46.994 \mathrm{db}$, and the average SSIM is 0.975 . The PSNR of the $12 \mathrm{~m}-264$ stream and the baseband signal is $47.291 \mathrm{db}$ and the ssim is 0.988 .

Experimental conclusion: The 264 signal encoded by Hunan Satellite TV has high image quality and low loss, the image quality will be improved slightly at $11 \mathrm{~m}$ rate. According to the characteristics of Hunan Satellite TV programs, it is generally dominated by variety shows, TV dramas and singing programs, which is very representative of the major satellite TV programs. The obtained data also objectively reflect the rate characteristics.

To sum up, the rate of live IPTV video is more reasonable at $2.5 \mathrm{Mbps}$, and the high definition can get high quality image quality in the case of 8 Mbps. If there are enough network resources, we can use 11-12 Mbps code to achieve better image quality in HD.

\section{References}

1 General Technical Requirements of IPTV service system [s]. 2008.

2. Gao xiaoneng, Guo Dachun. IPTV technical standards and application status [J ].Cheng technology and standardization, 2006,19 (3): 10-13. 